\title{
A Frequency Domain Robust Model Validation Approach to Fault Detection and Isolation with Applications to Identification of Contaminants in Lubrication and Transformer oils
}

\author{
Haftay Hailu \\ Department of Mechanical and Nuclear Engineering \\ The Pennsylvania State University \\ University Park, PA 16802
}

\author{
Mario Sznaier \\ Department of Electrical Engineering \\ The Pennsylvania State University \\ University Park, PA 16802
}

\begin{abstract}
This paper addresses the problem of robust fault detection and isolation in dynamical systems using frequency domain data. Our main result shows that this problem can be reduced to a convex optimization problem that can be efficiently solved. The proposed method is put to test on a nontrivial, practically relevant problem: detecting the presence and estimating the composition of contaminants in lubrication and transformer oil.
\end{abstract}

\section{INTRODUCTION}

$\mathrm{T}$ IE problem of fault detection and isolation in systems has been given considerable attention in recent years. The main drive for this is the growing need for on-board diagnostics and prognostics of systems in order to reduce maintenance costs and down time [1-3] focusing on condition based maintenance rather than schedule based maintenance. Many engineering systems such as airplane control systems, automotive electronic throttle and transmission systems, power distribution systems (such as transformers), chemical process control systems, high performance bearing systems, airborne systems and tanks equipped with gas turbines, all require the early fault detection in order to prevent catastrophic failure [3-8]. Some of the common classes of methods for fault detection and isolation include: model-based analytical methods, physical redundancy and model independent methods. Model based analytical approaches are popular due to their cost effectiveness. Many have studied this category using different mathematical models for the failure prediction [915]. However, the major challenge with the model-based approach is that it requires detailed knowledge of the physics of the system. Even in cases where a detailed model of the system is available, it may be difficult to exactly determine the values of all parameters involved, and any mismatch between the assumed and actual dynamics can lead to incorrectly concluding that faults are present. To address this difficulty, many researches have focused on robust FDI [12, 16-23]. This reduces the potential problem with model mismatch because it accounts for model uncertainty and measurement noise. To date, many of the

This work was supported in part by NSF under Grant DGE-0338240. work along these lines is based on the use of time-domain experimental data. However, in the case of the practical problem driving this paper, the experimental data available consists of measurements of the frequency response of the specimen, e.g. steady state values of the amplitude and phase shifts of sinusoidal inputs applied at several frequencies, and thus these methods are not exactly applicable. Motivated by this problem, in this paper we propose a frequency-domain counterpart of the time-domain robust FDI framework introduced in [22]. In principle this formulation leads a non-convex, computationally hard problem. However, as we show in the paper, a tight convex relaxation of the problem is readily available. These results are illustrated with a non-trivial practical problem arising in many industrial applications: identification and classification of contaminants in lubricating and transformer oils. The need for the close monitoring of lubricating / transformer oil conditions, in order to avoid the high cost of both preventive and corrective maintenance, has become more apparent recently. Small quantities of oil contaminants can cause serious system malfunctions and possibly hazardous situations. For example, glycol-water mixtures can hydrolyze the zinc dialkyldithiophosphate (ZDP) anti-wear additive [24] and if a significant amount of ZDP is so affected, anti-wear protection will be lost and catastrophic camshaft and valve lifter wear results. Similarly, a small amount electrolytic substance in transformer oils can lead to short circuit causing catastrophic malfunction of the transformer resulting in power interruptions and even worst fire hazard. Different researchers have proposed different methods of determining the condition of the oil. Some have used impedance methods along with equivalent circuit element representation of the electrochemical process [2426], while others have used oil conductivity as the measure of the oil condition [27]. The drawbacks of the above techniques are: 1) parametric fit is generally non-unique, for example there are many equivalent circuit-elementcombinations that give the same impedance curve, 2) uncertainty and measurement noise/disturbance are not addressed. Both effects make hard establishing that indeed contamination has taken place, and to ascertain the extent and composition of the contaminant. On the other hand, the 
technique proposed here can efficiently answer these questions.

The remainder of this paper is organized as follows: In Section II, we introduce the notation used in the paper. In section III we give a precise formulation of the problem under consideration, analyze its complexity and reduce it to a convex, Linear Matrix Inequality (LMI) optimization form. In Section IV, we illustrate these results with two examples, one academic and one practical. Finally, section $\mathrm{V}$ contains some conclusions and points out to directions for further research.

\section{PRELIMINARIES}

For ease of reference, next we summarize the notation used in this paper.

\begin{tabular}{|c|c|}
\hline $\bar{\sigma}(\mathbf{A})$ & Maximum singular value of $\mathbf{A}$ \\
\hline $\mathbf{A}>(\geq) 0$ & A is positive (semi) definite. \\
\hline $\mathbf{I}, \mathbf{0}$ & $\begin{array}{l}\text { The identity and zero matrices of } \\
\text { compatible dimensions (when } \\
\text { omitted). }\end{array}$ \\
\hline$D$ & Closed right half plane: $\{z: \operatorname{Re}(z) \geq 0\}$ \\
\hline$\partial D$ & $j \omega$ axis \\
\hline$L_{2}$ & $\begin{array}{l}\text { Extended Banach space of vector } \\
\text { valued real functions equipped with } \\
\text { the norm: }\|x\|_{2} \doteq\left(\int_{0}^{\infty}\|\mathbf{x}(t)\|_{2}^{2} d t\right)^{\frac{1}{2}}\end{array}$ \\
\hline$H_{\infty}$ & $\begin{array}{l}\text { Space of functions essentially } \\
\text { bounded on the } j \omega \text { axis, with } \\
\text { bounded analytic continuation inside } \\
\text { the right half plane, equipped with the } \\
\text { norm: }\|G\|_{\infty} \doteq \operatorname{ess} \sup _{\omega} \bar{\sigma}(G(j \omega)) \text {. }\end{array}$ \\
\hline$H_{\infty}$ & Closed unit ball in $H_{\infty}$. \\
\hline
\end{tabular}

\section{FREQUENCY DOMAIN BASED FDI}

\section{A. Problem Formulation}

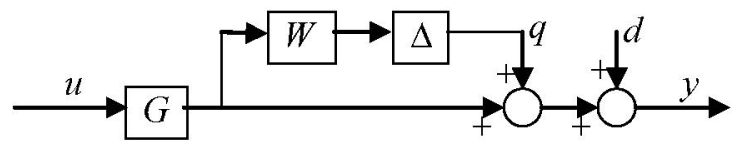

Figure 1. Setup for robust FDI with multiplicative uncertainty.

Consider the parameterized frequency domain model shown in Figure 1. The corresponding equation is given by:

$$
y=d+[I+W(s) \Delta(s)]\left[G_{0}(s)+\sum_{i=1}^{r} f_{i} G_{i}(s)\right] u
$$

where:
$G_{0}(s)$ is the model of the healthy plant

$G_{i}(s)$ represents the plant models corresponding to the $i^{\text {th }}$ fault.

$f_{i} \in[0,1]$ are fault indicators, i.e., $f_{i}=0$ (no fault), $f_{i}=1.0$ (extreme fault).

$u\left(j \omega_{k}\right), y\left(j \omega_{k}\right)$ and $d$ represent a known input, the corresponding frequency domain experimental output (response) and an unknown but $L_{2}$ bounded measurement noise, $\|d\|_{2} \leq \varepsilon$, respectively.

$W(s)$ is a known weighting function and $\Delta(s) \in \subseteq \bar{B} H_{\infty}$ represent the unstructured dynamic model uncertainty.

Using these definitions, the problem under consideration here can be precisely stated as: Given the following a priori information: a model of the plant under normal (healthy) conditions $G_{0}(s)$, a model of the possible failure modes $G_{i}(s)$, a bound $\varepsilon$ on the measurement noise, uncertainty and $N_{f}$ experimental input/output data pair $\left\{u\left(j \omega_{k}\right), y\left(j \omega_{k}\right)\right\}$, determine (i) whether a fault has occurred, and (ii) in this case, its location and strength. As we show next, this problem can be transformed to a (nonconvex) optimization form by exploiting the following boundary Nevanlinna-Pick interpolation result:

Lemma 1: Given $q_{k} \in \mathbb{C}^{m}, p_{k} \in \mathbb{C}^{n}$ and $z_{k} \in \partial D$, $k=1, \cdots, N_{f}$, there exist a matrix function $\Delta \in \bar{B} H_{\infty}$ such that

$$
q_{k}=\boldsymbol{\Delta}\left(z_{k}\right) p_{k}, \quad k=1, \cdots, N_{f}
$$

if and only if

$$
\left\|q_{k}\right\|_{2} \leq\left\|p_{k}\right\|_{2}, \quad k=1, \cdots, N_{f}
$$

Proof: The proof is given in [28].

Direct application of this Lemma leads to the following result:

Lemma 2: There exists a feasible triplet $\{\boldsymbol{\Delta}, \mathbf{f}, \mathbf{d}\}$ such that equation (1) holds if and only if the following set of inequalities is feasible:

$$
\begin{aligned}
& \begin{array}{l}
y_{k}^{*}\left(G u_{k}+d_{k}\right)+\left(G u_{k}+d_{k}\right)^{*} y_{k}-y_{k}^{*} y_{k}-\left(G u_{k}\right)^{*}\left(1-\gamma^{2} W\right)\left(G u_{k}\right) \\
\text { and, } \\
\qquad d_{k}^{*}\left(G u_{k}\right)+\left(G u_{k}\right)^{*} d_{k} \geq 0
\end{array} \\
& \qquad\left|d_{k}\right| \leq \varepsilon^{2}, \quad k=1, \ldots, N_{f}
\end{aligned}
$$


Proof: From Figure 1,

$$
\begin{aligned}
q_{k} & =y_{k}-G u_{k}-d \\
p_{k} & =W G u_{k}
\end{aligned}
$$

From Lemma 1, we have there exists $\Delta \in \boldsymbol{\Delta}$ mapping the input-output sequences $(p, q)$ if and only if

$$
\left\|q_{k}\right\|_{2}^{2} \leq \gamma^{2}\left\|p_{k}\right\|_{2}^{2}
$$

For our case the problem is SISO, hence (4) is written as

$$
\left|q_{k}\right|^{2} \leq \gamma^{2}\left|p_{k}\right|^{2}
$$

substituting (3) into (5)

$$
\begin{aligned}
\mid & \left|q_{k}\right|^{2} \leq \gamma^{2}\left|p_{k}\right|^{2} \\
\Leftrightarrow & q_{k}^{*} q_{k} \leq \gamma^{2} p_{k}^{*} p_{k} \\
\Leftrightarrow & {\left[y_{k}-d_{k}-G u_{k}\right]^{*}\left[y_{k}-d_{k}-G u_{k}\right]^{*} \leq \gamma^{2}\left(W G u_{k}\right)^{*}\left(W G u_{k}\right) } \\
\Leftrightarrow & y_{k}^{*}\left(G u_{k}+d_{k}\right)+\left(G u_{k}+d_{k}\right)^{*} y_{k}-y_{k}^{*} y_{k} \\
& \quad \quad\left(G u_{k}\right)^{*}\left(1-\gamma^{2} W\right)\left(G u_{k}\right)-\left(d_{k}^{*}\left(G u_{k}\right)+\left(G u_{k}\right)^{*} d_{k}\right) \geq 0
\end{aligned}
$$

This completes the proof.

Unfortunately, condition (2) above is bilinear in the variables $\left\{f_{i}, d_{k}\right\}$. It is well known that optimization problems involving these types of constraints are generically NP-hard (see for example [29]) and thus computationally expensive. To avoid this difficulty, in the sequel, motivated by the work in [22] we propose a convex relaxation of the problem that leads to computationally efficient solutions. To this effect, consider the block diagram shown in Figure 2, where the measurement noise has been moved ahead of the uncertainty block.

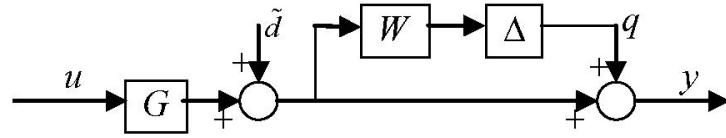

Figure 2. Setup for modified robust FDI with multiplicative uncertainty.

The corresponding equations are now given by

$$
y=[I+W(s) \Delta(s)]\left\{\left[G_{0}(s)+\sum_{i=1}^{r} f_{i} G_{i}(s)\right] u+d\right\}
$$

When compared to the original setup in Figure 1, it can be easily seen that the only difference is in the measurement noise level, since if there exists a triplet $\{\boldsymbol{\Lambda}, \mathbf{f}, \tilde{\mathbf{d}}\}$ satisfying equation (7) with $\|\tilde{\mathbf{d}}\|_{2} \leq \tilde{\varepsilon} \doteq \frac{\varepsilon}{1+\|W\|_{\infty}\|\Delta\|_{\infty}}$, then the triplet $\{\Delta, \mathbf{f}, \mathbf{d}\}$ with $\mathbf{d} \doteq(1+\Delta W) \tilde{\mathbf{d}}$ satisfies equation (1). It follows that one can attempt to find a solution to the original problem of Figure 1 by searching for a solution to the FDI problem shown in Figure 2, with noise level $\tilde{\varepsilon}$. As we show in the sequel this leads to a convex optimization problem. In addition, one will expect that if $\|W \Delta\| \ll 1$ then this approximation is not too conservative.

Remark 1: Note that in general, due to the presence of uncertainty and noise, there may exist more than one set $\{\Delta, \mathbf{f}, \mathbf{d}\}$ that explains the experimental input/output data. In that case, to avoid ambiguities, we will select among all possible solutions, the one corresponding to the minimum value of $\|\mathbf{f}\|_{2}$. This choice minimizes the number of false alarms, since it tries to explain, whenever possible, the experimental data as being produced by the normal (nonfailure) dynamics, possibly affected by dynamic uncertainty and measurement noise.

With this choice, the modified FDI problem can be recast into the following optimization form:

Problem 1: Given the a priori information $G_{i}(s), W(s), \varepsilon$ and the experimental data $\mathbf{u}$ and $\mathbf{y}$ find:

$$
\begin{aligned}
& \min _{\Delta, \mathbf{d}}\|\mathbf{f}\|_{2} \\
& \text { subject to: } \\
& \quad \Delta \in \bar{B} H_{\infty},\|\mathbf{d}\|_{2} \leq \varepsilon, \\
& y=[I+W(s) \Delta(s)]\left[\left[G_{0}(s)+\sum_{i=1}^{r} f_{i} G_{i}(s)\right] u+d\right\}
\end{aligned}
$$

If $\mathbf{f}=\mathbf{0}$ then no fault is present. Otherwise, the fault location/strength is identified by the elements of $\mathbf{f}$.

Theorem 1: Problem 1 is equivalent to the following LMI optimization problem:

$$
\begin{aligned}
& \min \|\mathbf{f}\|_{2} \\
& \text { subject to: } \\
& A_{k}\left(\tilde{d}_{k}, \mathbf{f}\right)= \\
& {\left[\begin{array}{rr}
y_{k}^{*}\left(G u_{k}+\tilde{d}_{k}\right)+\left(G u_{k}+\tilde{d}_{k}\right) y_{k}-y_{k}^{*} y_{k} & \left(G u_{k}+\tilde{d}_{k}\right) \\
\left(G u_{k}+\tilde{d}\right)^{*} & \left(1-\gamma^{2} W\right)^{-1}
\end{array}\right] \geq 0,} \\
& B_{k}\left(\tilde{d}_{k}\right)=\left[\begin{array}{cc}
\tilde{\varepsilon}^{2} & \tilde{d}_{k} \\
\tilde{d}_{k}^{\prime \prime} & 1
\end{array}\right] \geq 0, \quad k=1, \ldots, N_{f}
\end{aligned}
$$

Proof: From Figure 2,

$$
\begin{aligned}
& q_{k}=y_{k}-\left(G u_{k}+\tilde{d}\right) \\
& p_{k}=W\left(G u_{k}+\tilde{d}\right)
\end{aligned}
$$

substituting (10) into (5)

$$
\begin{aligned}
& \quad\left|q_{k}\right|^{2} \leq \gamma^{2}\left|p_{k}\right|^{2} \\
& \Leftrightarrow q_{k}^{*} q_{k} \leq \gamma^{2} p_{k}^{*} p_{k} \\
& \Leftrightarrow \quad\left[y_{k}-\left(G u_{k}+\tilde{d}_{k}\right)\right]^{*}\left[y_{k}-\left(G u_{k}+\tilde{d}_{k}\right)\right] \\
& \quad \leq \gamma^{2}\left[W\left(G u_{k}+\tilde{d}_{k}\right)\right]^{*}\left[W\left(G u_{k}+\tilde{d}_{k}\right)\right]
\end{aligned}
$$




$$
\begin{aligned}
\Leftrightarrow & y_{k}^{*}\left(G u_{k}+\tilde{d}_{k}\right)+\left(G u_{k}+\tilde{d}_{k}\right)^{*} y_{k}-y_{k}^{*} y_{k} \\
& -\left(G u_{k}+\tilde{d}_{k}\right)^{*}\left(1-\gamma^{2} W\right)\left(G u_{k}+\tilde{d}_{k}\right) \geq 0
\end{aligned}
$$

Using Schur complements the last inequality yields the first LMI in (9). The second LMI is just a restatement of $\left|\tilde{d}_{k}\right| \leq \tilde{\varepsilon}^{2}$.

This completes the proof.

\section{EXAMPLES: ANALYTICAL \& EXPERIMENTAL}

In this section we test the proposed technique using two FDI problems: one academic and one practical.

Example 1-Analytical Example: The analytical example selected for testing the current technique is a system and its three fault dynamics all subject to uncertainty, equation (11), where the healthy and faulty dynamic models are given by equation (12).

$$
\begin{aligned}
y & =(1+\Delta W)\left(G_{0}(s)+\sum_{i=1}^{3} f_{i} G_{i}(s)\right) u+d \\
G_{0} & =\frac{s^{4}-4.75 s^{3}-2.48 s^{2}-1.19 s-0.56}{s^{4}+1.92 s^{3}+1.61 s^{2}+0.83 s+0.16} \\
G_{1} & =\frac{5.07 s^{4}+3.91 s^{2}+0.94}{s^{4}+2.55 s^{3}+3.76 s^{2}+4.16 s+3.18} \\
G_{2} & =\frac{31.75 s^{3}+1.8 s}{s^{4}+2.55 s^{3}+3.76 s^{2}+4.16 s+3.18} \\
G_{3} & =\frac{75.75 s^{2}+65}{s^{4}+2.55 s^{3}+3.76 s^{2}+4.16 s+3.18}
\end{aligned}
$$

The frequency data was generated using the models and an assumed uncertainty and noise models. The magnitude plot of the models and the frequency data is shown in Fig. 3.

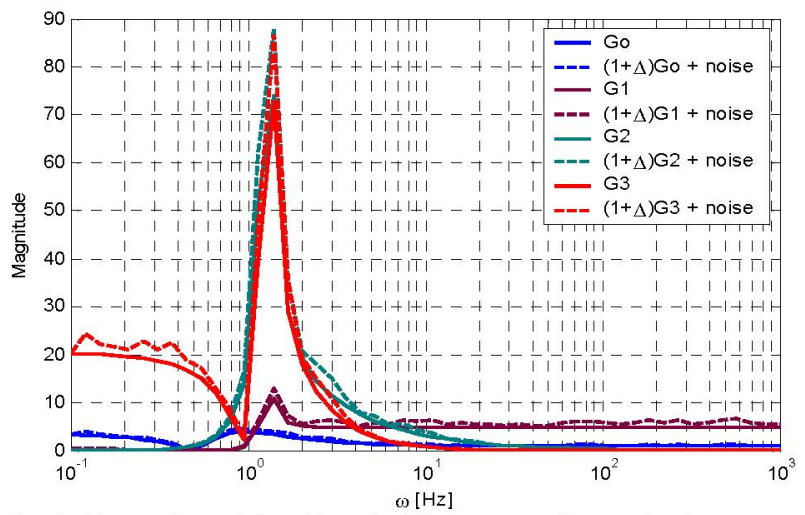

Fig. 3. Dynamic models with and without uncertainty and noise.

Table I shows the results of several experiments with simulated faults of different types and levels. The different simulated fault results show that the proposed technique correctly identified the type. It also provided a good estimate of level of the faults in each case.
TABLE I

RESULTS OF THE ANALYTICAL MODEL

\begin{tabular}{||ccc|ccc||}
\hline \hline \multicolumn{3}{|c|}{ Fault Mode } & \multicolumn{3}{c||}{ Detected Fault Strength } \\
\hline 0.0 & 0.0 & 0.0 & 0.0008 & 0.0008 & 0.0006 \\
\hline 0.0 & 0.0 & 1.0 & 0.0012 & 0.0007 & 0.8542 \\
\hline 0.0 & 1.0 & 0.0 & 0.0047 & 0.8404 & 0.0001 \\
\hline 0.0 & 1.0 & 1.0 & 0.0000 & 0.8453 & 0.8545 \\
\hline 1.0 & 0.0 & 0.0 & 0.8152 & 0.0027 & 0.0000 \\
\hline 1.0 & 0.0 & 1.0 & 0.7797 & 0.0003 & 0.8559 \\
\hline 1.0 & 1.0 & 0.0 & 0.7789 & 0.8415 & 0.0000 \\
\hline 1.0 & 1.0 & 1.0 & 0.7814 & 0.8455 & 0.8552 \\
\hline 0.2 & 0.7 & 0.9 & 0.1173 & 0.5852 & 0.7716 \\
\hline 0.01 & 0.95 & 0.42 & 0.0000 & 0.8007 & 0.3708 \\
\hline 0.48 & 0.56 & 0.67 & 0.3510 & 0.4765 & 0.5804 \\
\hline 0.1 & 0.3 & 0.2 & 0.0527 & 0.2588 & 0.1864 \\
\hline 0.37 & 0.66 & 0.84 & 0.2584 & 0.5564 & 0.7216 \\
\hline 0.33 & 0.33 & 0.33 & 0.2271 & 0.2887 & 0.2964 \\
\hline 0.85 & 0.75 & 0.95 & 0.6577 & 0.6364 & 0.8142 \\
\hline \hline
\end{tabular}

Example 2 - Experimental Example: In this example actual oil experimental data is used. The experimental frequency response data is obtained by interrogating oil samples using a small sinusoidal signal and measuring the steady state amplitude and phase. The experiment was conducted for 1) pure oil, 2) oil with contaminant-A, and 3) Oil with contaminant-B. A scaled magnitude plot of the data is shown in Figure 3. The dynamic models of the healthy plant and each failure mode were obtained using a leastsquares fitting of the corresponding frequency response. These models, for the healthy as well as the faulty dynamics are given by equation (13).

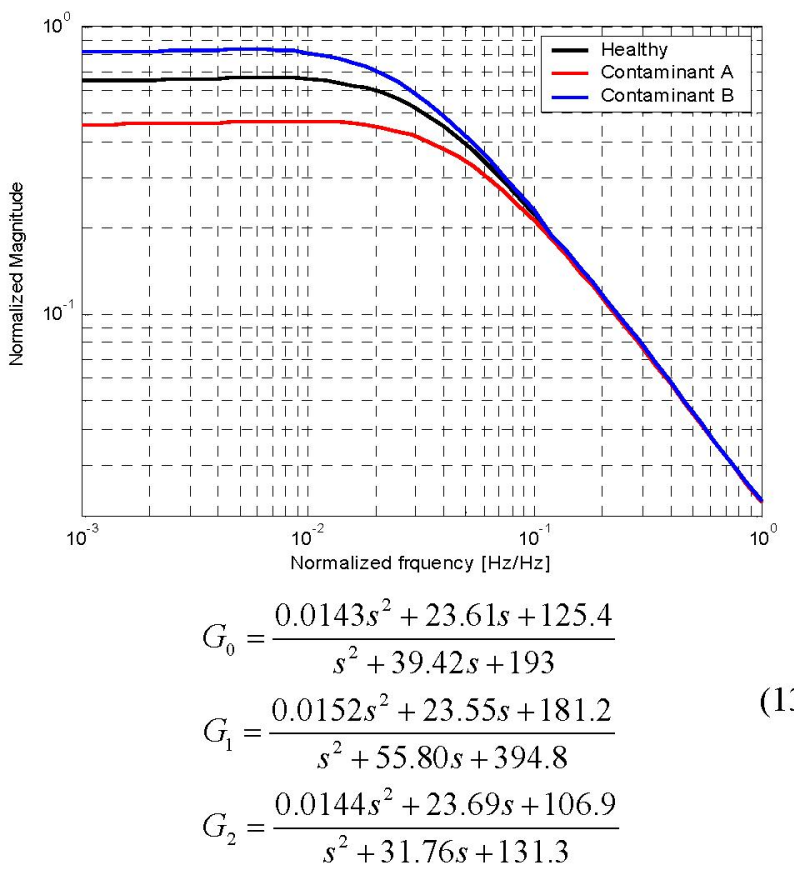


The proposed technique was tested using the models (13) and additional experimental data, not used in the identification. The results of applying the proposed FDI method to this new data are summarized in Table II. These results indicate that the developed method has effectively identified the fault types. The estimated fault levels are also generally good. In cases of single fault modes, the fault level was estimated to about $78-87 \%$ of the actual fault level.

TABLE II

RESULTS OF THE EXPERIMENTAL AND ANALYTICAL MODELS OF OIL

\begin{tabular}{||cc|cc||}
\hline \hline \multicolumn{2}{|c|}{ Fault Mode } & \multicolumn{2}{c||}{ Detected Fault Strength } \\
\hline \hline 0.0 & 0.0 & 0.0194 & 0.0382 \\
0.0 & 1.0 & 0.0000 & 0.7727 \\
1.0 & 0.0 & 0.8016 & 0.0000 \\
1.0 & 1.0 & 0.1131 & 0.0000 \\
0.2 & 0.7 & 0.0023 & 0.3417 \\
0.01 & 0.95 & 0.0018 & 0.7992 \\
0.48 & 0.56 & 0.0536 & 0.0505 \\
0.1 & 0.3 & 0.0037 & 0.1047 \\
0.33 & 0.33 & 0.0807 & 0.0466 \\
0.85 & 0.75 & 0.3471 & 0.1411 \\
\hline \hline
\end{tabular}

When simultaneous fault modes are present the estimation is lower than the single mode cases. This is because multiple contaminants do not necessarily have the same effect as the sum of the individual contaminants. Therefore, experiments should be performed for combination of multiple contaminants that are of practical interest. The response of such combination can be used along with the developed algorithm to identify the combination of the fault. Improving this estimation is practically significant because in most applications multiple contaminants (all at the same time) are more likely to exist than individual contaminants.

\section{CONCLusion}

In this paper we introduced a new robust FDI method based on frequency domain data. In principle this formulation leads to non-convex, generically NP hard problem. To circumvent this difficulty, we proposed a (tight) convex relaxation that allows for recasting the problem into an LMI optimization form. These results were illustrated with an academic example and a practical one, identifying the presence and composition of contaminants in oil. As shown in the paper, in both cases the proposed method was able to correctly identify faults, in the presence of model uncertainty and measurement noise. Research is currently under way to improve the estimation of the fault levels, by exploiting mixed time/frequency domain data, and to integrate the technique with robust model identification.

\section{REFERENCES}

[1] B. W. Wilson, N. H. Hansen, C. L. Shepard, T. J. Peters, and F. L. Greitzer, "Development of a Modular In-Situ Oil Analysis Prognostic System," presented at International Society of Logistics (SOLE) 1999 Symposium, Las Vegas, Nevada, 1999.

[2] S. You, M. Krage, and L. Jalics, "Overview of Remot Diagnostics and Maintenance for Automotive Systems," presented at 2005 SAE World Congress, Detroit, MI, 2005.

[3] F. L. Greitzer and R. A. Pawlowski, "Embedded Prognostics Health Monitoring," presented at International Instrumentation Symposium Embedded Health Monitoring Workshop, 2002

[4] R. Conaster, J. Waganer, S. Ganta, and I. Walker, "Diagnosis of Electronics Automotive Throttle Control Systems," Control Engineering Practice, vol. 12, pp. 23-30, 2004.

[5] M. W. Frye, R. M. Bailey, and A. D. Jessup, "NASA-Langley Research Center's Aircraft Condition Analysis and Management System Implementation," NASA, Hampton October 2004.

[6] L. Liu and D. A. Cartes, "On-line Identification and Robust Fault Diagnosis for Nonlinear PMSM Drives," presented at 2005 American Control Conference, Portland, OR, USA, 2005.

[7] A. G. Parlos, K. Kim, and R. M. Bharadwaj, "Sensorless Detection of Mechanical Faults in Electromechanical Systems," Mechatronics, vol. 14, pp. 357-380, 2004.

[8] X. Zhang, R. Xu, C. Kwan, S. Y. Liang, Q. Xie, and L. Haynes, "An Intergrated Approach to Bearing Fault Diagnostics and Prognostics," presented at 2005 American Control Conference, Portlan, OR, USA, 2005.

[9] R. J. Patton, "Fault detection and diagnosis in aerospace systems using analytical redundancy," Computing \& Control Engineering Journal, vol. 2, pp. 127, 1991.

[10] S. X. Ding, P. M. Frank, E. L. Ding, and T. Jeinsch, "Fault detection system design based on a new trade-off strategy," presented at Proceedings of the 39th IEEE Conference on Decision and Control, Sydney, Australia, 2000.

[11] D. Capriglione, C. Liguori, C. Pianese, and A. Pietrosanto, "On-line Sensor Fault Detection, Isolation and Accomodation in Automotive Engines," IEEE Transactions on Instrumentation and Measurement, vol. 52, pp. 1182-1189, 2003.

[12] P. M. Frank and X. Ding, "Survey of robust residual generation and evaluation in observer based fault detection systems," Journal of Process Control, vol. 7, pp. 403-429, 1997.

[13] J. J. Gertler, "Survey of model-based failure detection and isolation in complex plants," Control Systems Magazine, IEEE, vol. 8, pp. 3, 1988.

[14] R. Isermann and M. Ulieru, "Intergrated Fault Detection and Diagnosis," presented at IEEE/SMC"93 Conference on Systems Engineering in the Service of Humans, Le Touquet, France, 1993.

[15] H. Kirsch and K. Kroshel, "Applying Bayesian Networks to Fault Diagnosis," presented at 3rd IEEE Conference on Control Applications, 1994.

[16] E. Collins and T. Song, "Robust Hinfnity Estimation and Fault Detection of Uncertain Dynamic Systems," Journal of Guidance, Control and Dynamics, vol. 23, pp. 857-864, 2000.

[17] S. Duk-Sun and M. Sznaier, "A Caratheodory-Fejer approach to simultaneous fault detection and isolation," presented at American Control Conference, Denver, Colorado, 2003.

[18] A. Emami-Naeini, M. M. Akhter, and S. M. Rock, "Effect of Model Uncertainty on Fault Detection: The Threshold Selector," IEEE Transactions on Automatic Control, vol. 33, pp. 1106-1115, 1988.

[19] D. Henry, A. Zolghadri, F. Castang, and M. Monsion, "A New Multiobjective Filter Design for Guaranteed Robust FDI Performance," presented at Proceedings of the IEEE Conference on Decision and Control, 2001

[20] B. Jiang, J. Wang, and Y. Soh, "An Adaptive Technique for Robust Diagnostics of Faults with Independent Effects on System Outputs," International Journal of Control, vol. 75, pp. 782-802, 2002.

[21] A. Saberi, A. A. Stoorvogel, P. Sannuti, and H. Niemann, "Fundamental Problems in Fault Detection and Identification," International Journal of Robust and Nonlinear Control, vol. 10, pp. 1209-1236, 2000. 
[22] M. Sznaier, W. Ma, and C. Lagoa, "A Risk Adjusted Approach to Robust Simultaneous Fault Detection and Isolation," presented at 16th IFAC World Congress, Prague, Czech Republic, 2005.

[23] M. Zhong, S. X. Ding, J. Lam, and H. Wang, "An LMI Approach to Design Robust Fault Detection Filters for Uncertain LTI Systems," Automatica, vol. 39, pp. 543-550, 2003.

[24] S. S. Wang and H.-S. Lee, "The Application of A.C. Impedance Technique for Detecting Glycol Contamination in Engine Oil," Sensors and Actuators B, vol. 40, pp. 193-197, 1997.

[25] S. S. Wang, S. P. Maheswari, and S. C. Tung, "A.C. Impedance Measurements of the Resistance and Capacitance of Lubricants," ASLE Transaction, vol. 30, pp. 436-, 1987.

[26] A. P. Washabaugh, A. Mamishev, Y. Du, and M. Zahn, "Dielectric Measurements of Semi-insulating Liquids and Solids," presented at 12th International Confrence on Conduction and Breakdown in Dielectric Liquids, Roma, Italy, 1996.

[27] A. Basu, A. Berdorfer, C. Buelna, J. Cambell, K. Ismail, Y. Lin, L. Rodriquez, and S. S. Wang, "Smart Sensing of Oil Degradation and Oil Level Measurement in Gasoline Engines," presented at SAE 2000 World Congress, Detroit, Michigan, 2000.

[28] J. Chen and G. Gu, Control-Oriented System Identification: An $H_{\infty}$ Approach. New York: John Wiley \& Sons, 2000.

[29] H. D. Tuan and P. Apkarian, "Low Nonconvex Rank Bilinear Matrix Inequalities: Algorithms and Application," presented at 38th Conference on Decision and Control, Phoenix, AZ, USA, 1999. 